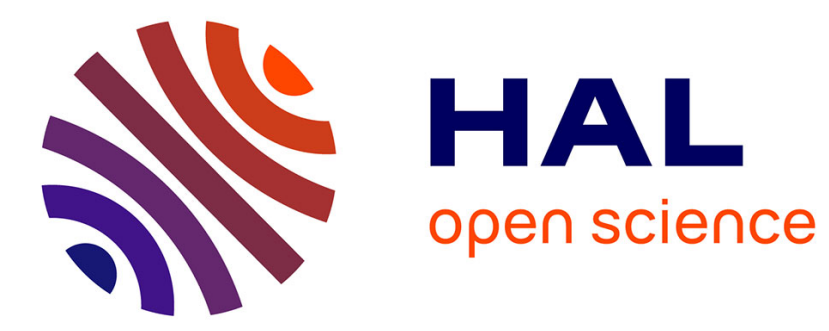

\title{
Synthèse et structure cristalline de $\beta-\mathrm{Cd} 2(\mathrm{OH}) 3 \mathrm{Cl}$ Yannick Cudennec, Yves Gerault, André Lecerf
}

\section{To cite this version:}

Yannick Cudennec, Yves Gerault, André Lecerf. Synthèse et structure cristalline de $\beta-\mathrm{Cd} 2(\mathrm{OH}) 3 \mathrm{Cl}$. Comptes rendus de l'Académie des sciences. Série IIb, Mécanique, 1997, 324 (7), pp.457-466. 10.1016/S1251-8069(99)80057-8 . hal-02947096

\section{HAL Id: hal-02947096 https://hal.science/hal-02947096}

Submitted on 23 Sep 2020

HAL is a multi-disciplinary open access archive for the deposit and dissemination of scientific research documents, whether they are published or not. The documents may come from teaching and research institutions in France or abroad, or from public or private research centers.
L'archive ouverte pluridisciplinaire HAL, est destinée au dépôt et à la diffusion de documents scientifiques de niveau recherche, publiés ou non, émanant des établissements d'enseignement et de recherche français ou étrangers, des laboratoires publics ou privés. 


\title{
Synthèse et structure cristalline de $\beta-\mathrm{Cd}_{2}(\mathrm{OH})_{3} \mathrm{Cl}$
}

\author{
Yannick CUDENNEC, Yves GERAULT et André LECERF
}

Laboratoire de Chimie des Matériaux Inorganiques et de Cristallographie, INSA-Rennes, 20, avenue des buttes de Coësmes, 35043 Rennes cedex, France.

Note présentée par Jean Rouxel.

https://doi.org/10.1016/S1251-8069(99)80057-8

Résumé. La structure cristalline de l'hydroxychlorure de cadmium $\beta-\mathrm{Cd}_{2}(\mathrm{OH})_{3} \mathrm{Cl}$ a été déterminée sur monocristal. La maille est orthorhombique de groupe spatial Pnma. Les paramètres cristallins sont les suivants : a $(\AA)=6,790(4), \mathrm{b}(\AA)=7,408(4), \mathrm{c}(\AA)=9,901(4), \mathrm{Z}=4, \rho$ calc $=$ $4,15 \mathrm{~g} \cdot \mathrm{cm}^{-3}$. La structure a été affinée jusqu'à un facteur résiduel final $\mathrm{R}=0,028(\mathrm{Rw}=0,055)$, en utilisant 476 réflexions indépendantes [ I > $3 \sigma$ ( I ) ]. La structure est isotype du composé correspondant du cuivre, l'atacamite $\mathrm{Cu}_{2}(\mathrm{OH})_{3} \mathrm{Cl}$. Elle est tridimensionnelle et peut être décrite comme un enchevêtrement croisé de chaînes d'octaèdres de cadmium $\left[\mathrm{Cd} \mathrm{O}_{4} \mathrm{Cl}_{2}\right]$ et $\left[\mathrm{Cd} \mathrm{O}_{5} \mathrm{Cl}\right]$, reliées entre elles.

\section{Synthesis and crystal structure of $\beta-\mathrm{Cd}_{2}(\mathrm{OH})_{3} \mathrm{Cl}$}

Abstract. The crystal structure of cadmium hydroxychloride $\beta-\mathrm{Cd}_{2}(\mathrm{OH})_{3} \mathrm{Cl}$ was determined with a single crystal. The unit-cell is orthorhombic, space grgup Pnma. Cell parameters are: a $(\AA)=$ $6,790(4), \mathrm{b}(\AA)=7,408(4), \mathrm{c}(\AA)=9,901(4), \mathrm{Z}=4, \rho_{\text {calc }}=4,15 \mathrm{~g} \cdot \mathrm{cm}^{-3}$. The structure was refined to the final reliability factor: $\mathrm{R}=0.028(\mathrm{Rw}=0.055)$, using 476 independent reflections $(\mathrm{I}>3 \sigma$ (I)). The crystal structure presents an isomorphism with atacamite $\mathrm{Cu}_{2}(\mathrm{OH})_{3} \mathrm{Cl}$. It has a threedimensional framework, built from intercrossing $\left[\mathrm{Cd} \mathrm{O}_{4} \mathrm{Cl}_{2}\right]$ and $\left[\mathrm{Cd} \mathrm{O}_{5} \mathrm{Cl}\right]$ octahedra chains.

\section{Abridged English version}

\section{Introduction}

Much work has been published on cadmium hydroxychlorides. The most important one was reported in 1910 [1]. Several crystallized phases are mentioned and have been studied: $\mathrm{Cd}(\mathrm{OH}) \mathrm{C} 1$, $\alpha$ and $\beta-\mathrm{Cd}_{2}(\mathrm{OH})_{3} \mathrm{Cl}$ and $\mathrm{Cd}(\mathrm{OH})_{(2-\mathrm{x})} \mathrm{Cl}_{\mathrm{x}}$. Only the crystal structure of $\mathrm{Cd}(\mathrm{OH}) \mathrm{C} 1$ has been determined from a single crystal [2]. Since 1970, few studies have been carried out on these compounds and, to our knowledge, no single crystal structure determination has been performed. We propose a new route for the synthesis of cadmium hydroxychlorides, which permits the 
formation of single crystals and the crystal structure determination of $\beta-\mathrm{Cd}_{2}(\mathrm{OH})_{3} \mathrm{Cl}$ discussed in this paper.

\section{Preparation}

In previous studies, starting materials used in syntheses were cadmium chloride and cadmium oxide, and under these conditions $\beta-\mathrm{Cd}_{2}(\mathrm{OH})_{3} \mathrm{Cl}$ could not been obtained. In our new synthesis, cadmium oxide is replaced by $\gamma-\mathrm{Cd}(\mathrm{OH})_{2}$. In three previous publications, we have shown that this solid phase is rather unstable at room temperature; its stability increases with the size of the crystals [14]-[16]. When the temperature increases, $\gamma-\mathrm{Cd}(\mathrm{OH})_{2}$ gives rise to $\beta-\mathrm{Cd}(\mathrm{OH})_{2}$, whose structure is isomorphous with brucite. It is this instability which is used in our new route. In aqueous solutions, in the presence of chloride ions, $\gamma-\mathrm{Cd}(\mathrm{OH})_{2}$, is transformed into cadmium hydroxychlorides instead of $\beta-\mathrm{Cd}(\mathrm{OH})_{2}$. The reactions are summarized in the following schemes:

$$
\begin{array}{ll}
\text { At } 323 \mathrm{~K} & \mathrm{Cd}^{2+}{ }_{(\mathrm{aq})}+2 \mathrm{Cl}_{(\mathrm{aq})}^{-}+\gamma-\mathrm{Cd}(\mathrm{OH})_{2(\mathrm{~s})} \rightarrow 2 \mathrm{Cd}(\mathrm{OH}) \mathrm{Cl}_{(\mathrm{s})} \\
\text { At } 343 \mathrm{~K} & \mathrm{Cd}^{2+}{ }_{(\mathrm{aq})}+2 \mathrm{Cl}_{(\mathrm{aq})}^{-}+3 \gamma-\mathrm{Cd}(\mathrm{OH})_{2(\mathrm{~s})} \longrightarrow 2 \alpha-\mathrm{Cd}_{2}(\mathrm{OH})_{3} \mathrm{Cl}_{(\mathrm{s})}
\end{array}
$$

For temperatures above $343 \mathrm{~K}, \alpha-\mathrm{Cd}_{2}(\mathrm{OH})_{3} \mathrm{Cl}_{(\mathrm{s})}$, is transformed into a non-stoichiometric phase, $\mathrm{Cd}(\mathrm{OH})_{(2-\mathrm{x})} \mathrm{Cl}_{\mathrm{x}}$, and a few $\beta-\mathrm{Cd}_{2}(\mathrm{OH})_{3} \mathrm{Cl}_{(\mathrm{s})}$ crystals. Owing to the large grain size of the starting material $\gamma-\mathrm{Cd}(\mathrm{OH})_{2}$, (around several tenths of a millimeter), the reaction kinetic is very slow and favors the formation of large crystals.

\section{Crystal structure determination}

The single crystal of $\beta-\mathrm{Cd}_{2}(\mathrm{OH})_{3} \mathrm{Cl}$ used for the crystallographic studies was colorless and had a pseudo-regular octahedral shape, with edges of around $0.2 \mathrm{~mm}$. The intensity data collection was performed with a MACH 3 NONIUS diffractometer operating with the Mo-K $\alpha$ radiation. The unitcell is orthorhombic, space group Pnma. The lattice parameters, a $(\AA)=6,790(4), \mathrm{b}(\AA)=7,408$ (4), c $(\AA)=9,901$ (4), were refined by least squares from 25 reflections. The Lorentz-polarisation correction has been applied to the data. The structural determination, carried out on a PC computer, using the XTAL software package confirmed straightforwardly the isomorphism of $\beta-\mathrm{Cd}_{2}(\mathrm{OH})_{3} \mathrm{Cl}$ with atacamite $\mathrm{Cu}_{2}(\mathrm{OH})_{3} \mathrm{Cl}$ [7]. Experimental conditions of the crystal study and results of the structural refinement are reported in table I, atomic position parameters, in table II.

\section{Discussion}

The structure has a three-dimensional framework, derived from the $\mathrm{NaCl}$ type, in which half the cation sites are empty [8]. The consequence is the formation of tunnels parallel to the $\mathbf{a}$ and $\mathbf{b}$ axes, as shown in figures 1 and 2. Coordinations around $\mathrm{Cd}, \mathrm{Cl}$ and $\mathrm{O}$ atoms are shown in figure 3, bond distances and angles are reported in table III.

The $\left[\mathrm{Cd}(1) \mathrm{O}_{4} \mathrm{Cl}_{2}\right]$ octahedra are linked together via two $(\mathrm{O}(1)-\mathrm{Cl})$ edges to form chains in the $\mathbf{b}$ direction, whereas the $\left[\mathrm{Cd}(2) \mathrm{O}_{5} \mathrm{Cl}\right]$ octahedra form chains in the a direction via two $(\mathrm{O}(2)-\mathrm{O}(2))$ edges. These two types of chains are linked together through $(\mathrm{O}(1)-\mathrm{O}(2))$ and $(\mathrm{O}(2)-\mathrm{C} 1)$ edges. The structure can be described as cross-linked chains giving rise to perpendicular tunnels. In the b direction, tunnels are narrow because of the presence of chloride atoms (fig. 2).On the other hand, in the a direction they are wider since only hydrogen atoms are present (fig. 1). The crystal structure of atacamite has a strong stability which is probably due to the Jahn-Teller effect of divalent copper. 
This effect explains why, generally, divalent copper crystallizes in its own particular structures. Therefore, the isomorphism of $\beta-\mathrm{Cd}_{2}(\mathrm{OH})_{3} \mathrm{Cl}$ with atacamite is rather surprising.

\section{Introduction}

L'étude des hydroxychlorures de cadmium a fait l'objet de nombreux travaux dont les plus importants ont été rapportés dans une publication de 1970 [1], qui faisait le point sur ces composés. L'existence de plusieurs phases cristallisées, annoncées antérieurement, y est confirmée par des analyses chimiques, thermiques, infrarouges et radiocristallographiques. Quatre hydroxychlorures de cadmium ont été étudiés : $\mathrm{Cd}(\mathrm{OH}) \mathrm{C} 1, \mathrm{Cd}_{2}(\mathrm{OH})_{3} \mathrm{Cl} \alpha$ et $\beta$ ainsi qu'une phase de stoechiométrie variable $\mathrm{Cd}(\mathrm{OH})_{(2-\mathrm{x})} \mathrm{Cl}_{\mathrm{x}}$. La structure cristalline de $\mathrm{Cd}(\mathrm{OH}) \mathrm{Cl}$ a été déterminée sur monocristal en 1934[2]. Une hypothèse de maille pour $\alpha-\mathrm{Cd}_{2}(\mathrm{OH})_{3} \mathrm{Cl}$ a été proposée sur la base du diagramme de poudre, et une isotypie avec 1'atacamite $\mathrm{Cu}_{2}(\mathrm{OH})_{3} \mathrm{Cl}$ a été avancée pour la variété $\beta-\mathrm{Cd} 2(\mathrm{OH})_{3} \mathrm{Cl}$ [1]. Quant à la phase solide $\mathrm{Cd}(\mathrm{OH})_{(2-x)} \mathrm{C} 1_{x}$, une maille cristalline a été proposée en 1945 [3].

Depuis 1970, peu d'études ont été entreprises sur ce sujet et, à notre connaissance, aucune étude cristallographique n'a été publiée. Il n'existe donc pas de données structurales complètes et fiables sur les hydroxychlorures de cadmium, en dehors de la structure de $\mathrm{Cd}(\mathrm{OH}) \mathrm{Cl}$ déterminée sur un monocristal, mais en 1934.

La mise au point d'une nouvelle voie de synthèse des hydroxychlorures de cadmium, nous a permis de préparer des monocristaux et notamment ceux de la variété $\beta-\mathrm{Cd}_{2}(\mathrm{OH})_{3} \mathrm{Cl}$, ce qui a rendu possible sa détermination structurale faisant l'objet de cette publication.

\section{Synthèse et préparation des monocristaux}

La méthode classique de synthèse utilisée pour la préparation des hydroxychlorures de cadmium [1], consiste à faire réagir le chlorure et l'oxyde de cadmium en milieu aqueux, à des températures allant de la température ambiante jusqu'à $473 \mathrm{~K}$. Pour la phase $\beta-\mathrm{Cd}_{2}(\mathrm{OH})_{3} \mathrm{Cl}$, sa préparation directe n'a pas été possible. Elle est obtenue par déplacement de l'iode contenu dans la phase $\beta-\mathrm{Cd}_{2}(\mathrm{OH})_{3} \mathrm{I}$ [1], par le chlore.

La nouvelle voie de synthèse que nous avons mise au point, consiste à utiliser non plus l'oxyde de cadmium, mais la variété $\gamma$ de l'hydroxyde: $\gamma-\mathrm{Cd}(\mathrm{OH})_{2}$. Dans trois publications antérieures [4-6], nous avons montré que cette variété était métastable à température ambiante; sa stabilisation étant essentiellement due à la taille élevée de ses grains. En milieu aqueux, lorsque la température augmente, elle se transforme en la variété stable thermodynamiquement, $\beta-\mathrm{Cd}(\mathrm{OH})_{2}$, dont la structure est de type brucite. C'est cette instabilité qui a été mise à profit dans cette nouvelle voie de synthèse. En présence d'ions chlorures introduits par l'intermédiaire du chlorure de cadmium hydraté très soluble, ce n'est plus $\beta-\mathrm{Cd}(\mathrm{OH})_{2}$ qui se forme, mais des hydroxychlorures de cadmium. Toutes les phases solides mises en évidence ont été caractérisées par leur diagramme de poudre obtenu sur un diffractomètre de poudre RIGAKU D-MAX II, grâce à la radiation $\mathrm{Cu}-\mathrm{K} \alpha$.

Si l'on introduit 1 mole de $\mathrm{CdCl}_{2}, 2,5 \mathrm{H}_{2} \mathrm{O}$ pour 1 mole de $\gamma-\mathrm{Cd}(\mathrm{OH})_{2}$, il se forme $\mathrm{Cd}(\mathrm{OH}) \mathrm{C} 1$ dès $323 \mathrm{~K}$, selon le schéma réactionnel suivant :

$$
\mathrm{Cd}^{2+}{ }_{(\mathrm{aq})}+2 \mathrm{Cl}_{(\mathrm{aq})}^{-}+\gamma-\mathrm{Cd}(\mathrm{OH})_{2(\mathrm{~s})} \longrightarrow 2 \mathrm{Cd}(\mathrm{OH}) \mathrm{Cl}_{(\mathrm{s})}
$$

En revanche, si le rapport est de 1 mole de chlorure de cadmium pour 3 moles d'hydroxyde, la réaction donne naissance à un mélange d'hydroxychlorures dont la composition varie avec la température. A $343 \mathrm{~K}$, on obtient un mélange contenant essentiellement la phase $\alpha-\mathrm{Cd}_{2}(\mathrm{OH})_{3} \mathrm{Cl}$ : 


$$
\mathrm{Cd}_{(\mathrm{aq})}^{2+}+2 \mathrm{Cl}_{(\mathrm{aq})}^{-}+3 \gamma-\mathrm{Cd}(\mathrm{OH})_{2(\mathrm{~s})} \longrightarrow 2 \alpha-\mathrm{Cd}_{2}(\mathrm{OH})_{3} \mathrm{Cl}_{(\mathrm{s})}
$$

La phase $\alpha$ n'est pas pure car elle contient un peu de phase de stoechiométrie variable $\mathrm{Cd}(\mathrm{OH})_{(2-\mathrm{x})} \mathrm{C} 1_{\mathrm{x}}$. Si l'on porte le système à une température proche de $373 \mathrm{~K}$, le mélange solide obtenu est triphasé. Il contient essentiellement cette dernière phase $\mathrm{Cd}(\mathrm{OH})_{(2-\mathrm{x})} \mathrm{C} 1_{\mathrm{x}}$, en coexistence avec un peu de variété $\alpha$ résiduelle et des cristaux de la variété $\beta-\mathrm{Cd}_{2}(\mathrm{OH})_{3} \mathrm{Cl}$. Il semble donc que la variété $\alpha-\mathrm{Cd}_{2}(\mathrm{OH})_{3} \mathrm{Cl}$ soit une phase de plus basse température que la variété $\beta$. De plus la transformation $\alpha \rightarrow \beta$ sous l'influence de la température est concurrencée par la formation de la phase de stoechiométrie variable, sans doute plus stable à température plus élevée. Cela explique peut être pourquoi la phase $\beta$ n'a jamais été mise en évidence directement dans les précédents travaux; son obtention nécessitant le passage par le composé iodé $\beta-\mathrm{Cd}_{2}(\mathrm{OH})_{3} \mathrm{I}$.

En outre, le fait de disposer d'un hydroxyde de cadmium $\gamma-\mathrm{Cd}(\mathrm{OH})_{2}$, dont la taille de grain est élevée (cristaux de plusieurs dixièmes de millimètre), permet de freiner considérablement les réactions de formation des hydroxychlorures, ce qui est très favorable à l'obtention de monocristaux. Les premiers essais entrepris ont été fructueux puisque des monocristaux de la variété $\beta-\mathrm{Cd}_{2}(\mathrm{OH})_{3} \mathrm{Cl}$ ont été obtenus; leur taille importante permettant de les stabiliser. Un système réactionnel de composition : 0,1 mole de $\mathrm{CdCl}_{2}, 2,5 \mathrm{H}_{2} \mathrm{O}, 0,3$ mole de $\gamma-\mathrm{Cd}(\mathrm{OH})_{2}$, pour 100 moles d'eau, est placé pendant une semaine, dans une étuve dont la température est augmentée progressivement jusqu'à $373 \mathrm{~K}$. Le système n'étant pas parfaitement étanche, une évaporation partielle se produit. Dans le mélange obtenu prennent naissance des cristaux sous la forme d'octaèdres incolores et transparents, peu déformés, dont l'arête a une taille moyenne de 0,2-0,3 $\mathrm{mm}$. Après filtration, lavage à l'eau et séchage sous vide sur potasse, quelques cristaux ont été prélevés, et introduits dans un verre de lindemann. Un diagramme de poudre effectué grâce à une chambre de Gandolfi, avec la radiation $\mathrm{K} \alpha$ du cuivre montre sans ambiguïté qu'il s'agit bien de la phase $\beta-\mathrm{Cd}_{2}(\mathrm{OH})_{3} \mathrm{Cl}$, dont le diagramme $\mathrm{X}$ est connu [1].

\section{Détermination de la structure}

Le monocristal choisi est un octaèdre transparent. presque régulier, dont les diagonales ont une taille voisine de $0,2 \mathrm{~mm}$. La mesure des intensités de diffraction a été réalisée grâce à un diffractomètre MACH 3 NONIUS, utilisant la longueur d'onde Mo-K $\alpha$, monochromatisée par une lame de graphite. La maille cristalline est orthorhombique. Les paramètres a $(\AA)=6,790$ (4), b $(\AA)$ $=7,408(4)$, c $(\AA)=9,901$ (4) ont été affinés par moindres carrés, à l'aide de 25 réflexions optimisées. L'étude structurale a été conduite sur PC, grâce à la chaîne de programmes XTAL. L'hypothèse d'une isotypie avec l'atacamite $\mathrm{Cu}_{2}(\mathrm{OH})_{3} \mathrm{Cl}$ [7], de groupe spatial Pnma, est alors vérifiée. A partir des positions atomiques de ]'atacamite, l'affinement de la structure converge très rapidement jusqu'à une valeur de $\mathrm{R}$ égale à $3,7 \%\left(\mathrm{R}_{\mathrm{w}}=3,2 \%\right)$. Après des corrections d'absorption effectuées grâce au programme Difabs, l'affinement de la structure conduit à une valeur de $\mathrm{R}$ égale à $2,8 \%\left(\mathrm{R}_{\mathrm{w}}=5,5 \%\right)$. Les conditions d'enregistrement ainsi que les résultats de l'affinement de la structure sont reportés dans le tableau $I$.

La structure de $\beta-\mathrm{Cd}_{2}(\mathrm{OH})_{3} \mathrm{Cl}$ comporte deux cadmiums, un chlore, deux oxygènes et deux hydrogènes, indépendants. L'affinement des positions atomiques ainsi que des paramètres d'agitation thermique anisotrope n'a pas été effectué pour les atomes d'hydrogène; leurs positions ayant été déterminées à partir de celles de l'hydrogène de l'atacamite, en respectant les distances et angles de liaisons caractérisant ce type d'atome. Les paramètres de positions atomiques sont reportés dans le tableau II. 


\section{Discussion}

La structure est tridimensionnelle et dérive de la structure de type $\mathrm{NaCl}$, dans laquelle la moitié des sites cationiques ne seraient pas occupés [8], ce qui se traduit par la formation de tunnels se développant suivant les axes a et $\mathbf{b}$. Cette caractéristique de la structure apparaît bien sur les figures 1 et 2, obtenues grâce au logiciel de dessin de structures cristallines CaRIne Cristallographie 3.0. Le cadmium $\mathrm{Cd}(1)$ est entouré par quatre oxygènes à une distance moyenne de 2,243 $\AA$. Deux chlores situés à 2,829 Å, complètent son environnement octaédrique peu déformé si l'on excepte les deux liaisons longues avec le chlore, pour donner le polyèdre $\mathrm{Cd} 1(\mathrm{O} 1)_{2}\left(\mathrm{O}_{2}\right)_{2}(\mathrm{Cl})_{2}$. L'environnement octaédrique du cadmium $\mathrm{Cd}(2)$ est plus déformé. Il comprend cinq oxygènes à une distance moyenne de 2,272 $\AA$; un chlore à $2,735 \AA$ complétant le polyèdre $\mathrm{Cd} 2(\mathrm{O} 2)_{2}\left(\mathrm{O}_{2}{ }^{\prime}\right)_{2}(\mathrm{O} 1)(\mathrm{Cl})$. Le chlore se trouve dans un environnement constitué par trois cadmiums $(2 \mathrm{Cd} 1+1 \mathrm{Cd} 2)$ et trois oxygènes $(1 \mathrm{O} 1+2 \mathrm{O} 2)$, formant une bipyramide à bases triangulaires en positions superposées. Enfin les atomes d'oxygène se trouvent dans un environnement tétraédrique constitué par trois cadmiums et un hydrogène $(2 \mathrm{Cd} 1+1 \mathrm{Cd} 2+\mathrm{H} 1$ pour $\mathrm{O} 1)$; $(1 \mathrm{Cd} 1+2 \mathrm{Cd} 2+\mathrm{H} 2$ pour $\mathrm{O} 2)$. Les distances et angles interatomiques sont reportés dans le tableau III. Les environnements autour de chaque atome sont représentés sur la figure 3. Les polyèdres de cadmium $\mathrm{Cd} 1$ mettent en commun deux arêtes définies par $(\mathrm{Ol}-\mathrm{Cl})$ pour former des chaînes qui se développent suivant 1a direction $\mathbf{b}$, en revanche les polyèdres de cadmium $\mathrm{Cd} 2$ s'enchaînent dans la direction a par deux arêtes définies par $(\mathrm{O} 2-\mathrm{O} 2)$ et $\left(\mathrm{O}_{2}^{\prime}-\mathrm{O} 2 '\right)$. La liaison entre les chaînes est assurée par la mise en commun entre des polyèdres $\mathrm{Cd} 1$ et $\mathrm{Cd} 2$ d'arêtes définies par $\left(\mathrm{O}_{1}-\mathrm{O}_{2}\right)$ et $\left(\mathrm{O}_{2}-\mathrm{Cl}\right)$. La structure tridimensionnelle obtenue peut donc être décrite comme l'enchevêtrement croisé de deux types de chaînes reliées entre elles, générant des tunnels dans les directions $\mathbf{a}$ et $\mathbf{b}$. Dans la direction $\mathbf{b}$, ces tunnels sont très étroits du fait de la présence des atomes de chlore (fig.2). En revanche, dans la direction a, leur volume est plus important (fig.1), car ils accueillent les hydrogènes. Cette occupation de lacunes cationiques par plusieurs hydrogènes est un phénomène déjà mentionné dans plusieurs publications [9-12], relatives aux composés $\mathrm{Ca}_{3} \mathrm{Al}_{2} \mathrm{Si}_{3} \mathrm{O}_{12}$ et $\mathrm{Ca}_{3} \mathrm{Al}_{2}(\mathrm{OH})_{12}$, et relative [13] au composé $\mathrm{Na}_{2} \mathrm{Cu}(\mathrm{OH})_{4}$. Les liaisons hydrogènes déjà faibles dans l'atacamite sont pratiquement inexistantes dans $\beta-\mathrm{Cd}_{2}(\mathrm{OH})_{3} \mathrm{Cl}$, si l'on compare les distances $\mathrm{O} 1-\mathrm{Cl}$ et $\mathrm{O} 2-\mathrm{Cl}$, respectivement 3,044 et 3,075 pour l'atacamite; et 3,495 et $3,421 \AA$ pour le composé du cadmium. Néanmoins par analogie avec l'atacamite, nous avons fait apparaître les atomes concernés par ces liaisons dans les environnements des atomes de cadmium, de chlore et d'oxygène (fig. 3).

La détermination structurale de $\beta-\mathrm{Cd}_{2}(\mathrm{OH})_{3} \mathrm{Cl}$, sur monocristal apporte des données cristallographiques fiables sur les hydroxychlorures de cadmium, puisque seule la structure de $\mathrm{CdOHCl}$ avait été déterminée, mais en 1934. La structure de $\beta-\mathrm{Cd}_{2}(\mathrm{OH})_{3} \mathrm{Cl}$ est isotype de celle de l'atacamite $\mathrm{Cu}_{2}(\mathrm{OH})_{3} \mathrm{Cl}$, principal constituant des mines de cuivre du désert d'Atacama au Chili. L'isotypie constatée avec le composé de même stoechiométrie du cuivre est assez originale. En effet, le cuivre divalent induit le plus souvent des structures qui lui sont particulières à cause de l'effet Jahn-Teller important qu'il présente. Dans la structure de l'atacamite, cet effet peut être mis en évidence en comparant les valeurs des longueurs de liaisons purement ioniques, obtenues grâce à la somme des rayons ioniques des ions dans un environnement octaédrique, avec les valeurs expérimentales.

$$
. \mathrm{R} \mathrm{Cu}^{2+}=0,72 \AA ; \cdot \mathrm{R} \mathrm{Cd}^{2+}=0,97 \AA ; \mathrm{R} \mathrm{O}^{2-}=1,40 \AA ; \mathrm{R} \mathrm{Cl}^{-}=1,81 \AA \text { [14] }
$$

$\mathrm{d}\left(\mathrm{Cu}^{2+}-\mathrm{O}^{2-}\right)=2,12 \AA$ (moyenne expérimentale 4 courtes : $1,99 \AA$, longue: $2,358 \AA$ )

$\mathrm{d}\left(\mathrm{Cu}^{2+}-\mathrm{Cl}^{-}\right)=2,53 \AA$ ( moyenne expérimentale: $2,76 \AA$ ) 
Le cadmium divalent, bien évidemment, ne présente pas d'effet Jahn-Teller.

$$
\begin{aligned}
& \mathrm{d}\left(\mathrm{Cd}^{2+}-\mathrm{O}^{2-}\right)=2,37 \AA \quad(\text { moyenne expérimentale: } 2,26 \AA) \\
& \left.\mathrm{d}\left(\mathrm{Cd}^{2+}-\mathrm{Cl}^{-}\right)=2,78 \AA \text { ( moyenne expérimentale: } 2,80 \AA\right)
\end{aligned}
$$

La structure de l'atacamite est très stable. Cette stabilisation est vraisemblablement due à l'effet Jahn-Teller important du cuivre divalent. En revanche, la structure de $\beta-\mathrm{Cd}_{2}(\mathrm{OH})_{3} \mathrm{Cl}$ est beaucoup moins stable. Cette phase apparaît en effet de façon transitoire lors de la transformation de $\alpha$ $\mathrm{Cd}_{2}(\mathrm{OH})_{3} \mathrm{Cl}$ en $\mathrm{Cd}(\mathrm{OH})_{(2-\mathrm{x})} \mathrm{Cl}_{\mathrm{x}}$, sous l'influence de la température. Sa préparation n'est possible que dans des conditions de synthèse qui permettent l'obtention de cristaux de taille importante, ce qui a pour conséquence de les stabiliser. La nouvelle méthode de synthèse utilisant $\gamma-\mathrm{Cd}(\mathrm{OH})_{2}$ comme matière de départ nous incite à penser que des monocristaux des autres hydroxychlorures peuvent être obtenus. Il est bon de rappeler que l'étude de ces composés n'est pas sans intérêt pour 1a compréhension des processus de dissolution de l'oxyde ou de l'hydroxyde de cadmium, en présence de chlorure; ces composés constituant la matière active anodique de l'accumulateur au nickelcadmium.

Note remise le 3 février 1997, acceptée après révision le 2 février 1997.

\section{Références bibliographiques}

[1] Walter-Lévy L., Groult D., 1970. Contribution à l'étude des halogénures basiques de cadmium, I, Sur la formation et les propriétés des chlorures basiques de cadmium, Bull. Soc. Chim. Fr., 11, p. 3868-3878.

[2] Hoard J. L., Grenko J. D., 1934. The crystal structure of cadmium hydroxychloride CdOHCl, Z. Krist., 87, p. 110-119.

[3] Feitknecht W., 1945. Experientia, 1, p. 230, cité dans [1].

[4] Lecerf A., Riou A., Cudennec Y., Gérault Y., Chanson C., 1988. Étude physico-chimique et structurale de l'hydroxyde de cadmium $\gamma-\mathrm{Cd}(\mathrm{OH})_{2}$, Mater. Res. Bull., 23, p. 1479-1490.

[5] Cudennec Y., Lecerf A., Riou A., Gérault Y., 1989. Synthèse et étude de l'hydroxycadmiate de sodium : intermédiaire réactionnel pour la préparation $\gamma-\mathrm{Cd}(\mathrm{OH})_{2}$, Mat. Res. Bull., 24, p. 381-388.

[6] Riou A., Cudennec Y., Gérault Y., 1990. Étude structurale de $\gamma-\mathrm{Cd}(\mathrm{OH})_{2}$ ou $\mathrm{Cd}_{2} \mathrm{O}(\mathrm{OH})_{2}\left(\mathrm{H}_{2} \mathrm{O}\right)$, Mat. Res. Bull., 25 , p. 987-996.

[7] Parise J. B., Hyde B. G., 1986. The structure of atacamite and its relationship to spinel, Acta Cryst.,C42, p. 1277-1280.

[8] Wells A. F., 1975. Structural inorganic chemistry, p. 142-143 in Structural inorg. Chem., 4th ed., Oxford Clarendon Press.

[9] McConnell D., Verhoek F. H., 1963. J. Chem. Educ., 40, p. 512-515, cité dans [7].

[10] Cohen-Addad C., Ducros P., Bertaut E. F, 1967. Acta Cryst., 23, p. 220-230, cité dans [7].

[11] Cohen-Addad C., Ducros P., Durif A., Bertaut E. F., Delapalme A., 1964. J. Phys. (Paris), 25, p. 478-483, cité dans [7].

[12] Foreman D. W., 1968. J. Chem. Phys., 48, p. 3037-3041, cité dans [7].

[13] Riou A., Cudennec Y., Gérault Y., 1989. Copper disodium tetrahydroxide, Acta Cryst., C45, p. 374-376.

[14] Ahrens L. H., 1952. Ionic radius table, Geochim. Cosmochim. Acta, 2, p. 155. 
Données expérimentales pour l'étude structurale.

Experimental data for the crystal study.

Données cristallines

Taille du cristal

Symétrie

Groupe spatial

Paramètres maille $(\AA)$

Volume $\left(\AA^{3}\right)$

Masse molaire

Z

Masse volumique

Mesures des intensités

\section{$\lambda \mathrm{Mo}-\mathrm{K} \alpha$}

Monochromateur

Mode de balayage

Amplitude de balayage

Ouverture de fente

$\theta$ maximal

$h k l$ (min., max.)

Réflexions standards

Réflexions mesurées

Réflexions indépendantes

Réflexions : $I>3 \sigma(I)$

Coefficient d'absorption $(\mu)$

Coefficient de transmission min.

Coefficient de transmission max.

\section{Affinement}

Paramètres affinés

Facteurs $R, R_{w}$

Esd

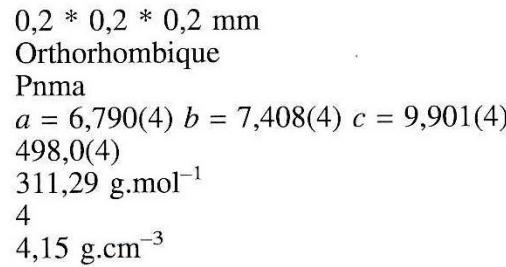

$0,71073 \AA$

Lame de graphite

$\omega-2 \theta$

$1,13+0,74 \tan \theta$

$2,01+2,24 \tan \theta$

$30^{\circ}$

$h(0,9) k(0,10) l(-13,13)$

3 , mesure toutes les $2 \mathrm{~h}$, pas de variations

1656

880

476

$89,381 \mathrm{~cm}^{-1}$

0,8814

1,1681

34

0,$028 ; 0,055$

1,429

TABLEAU II

Coordonnées atomiques ; les positions des atomes $\mathrm{H}$ n'ont pas été affinées.

Atomic coordinates; $H$ atom positions were not refined.

\begin{tabular}{|c|c|c|c|c|c|}
\hline Atomes & $x$ & \multicolumn{2}{|c|}{$y$} & $z$ & Ueq $\times 100\left(\AA^{2}\right)$ \\
\hline $\mathrm{Cd}(1) 4 \mathrm{a}$ & 0,0000 & \multicolumn{2}{|c|}{0,0000} & 0,0000 & $1,98(3)$ \\
\hline $\mathrm{Cd}(2) 4 \mathrm{c}$ & $0,1919(2)$ & \multicolumn{2}{|c|}{0,2500} & $0,2607(1)$ & $1,74(3)$ \\
\hline $\mathrm{Cl} \quad 4 \mathrm{c}$ & $0,3104(8)$ & \multicolumn{2}{|c|}{0,7500} & $0,0369(4)$ & $2,1(1)$ \\
\hline$O(1) 4 c$ & $0,183(2)$ & \multicolumn{2}{|c|}{0,2500} & $0,026(1)$ & $1,5(3)$ \\
\hline $\mathrm{O}(2) 8 \mathrm{~d}$ & $0,443(1)$ & \multicolumn{2}{|c|}{$0,054(1)$} & $0,2808(8)$ & $1,9(2)$ \\
\hline $\mathrm{H}(1) 4 \mathrm{c}$ & 0,305 & \multicolumn{2}{|c|}{0,250} & 0,990 & 3,5 \\
\hline$H(2) 8 d$ & 0,430 & \multicolumn{2}{|c|}{0,960} & 0,220 & 3,5 \\
\hline \multicolumn{5}{|c|}{$U_{(i, j)}^{*} \times 100$} & \\
\hline$U(1,1)$ & $U(2,2)$ & $U(3,3)$ & $U(1,2)$ & $U(1,3)$ & $U(2,3)$ \\
\hline $2,43(5)$ & $1,69(5)$ & $1,83(5)$ & $-0,27(6)$ & $0,20(5)$ & $-0,59(5)$ \\
\hline $1,29(4)$ & $2,01(4)$ & $1,92(5)$ & 0,00 & $-0,42(6)$ & 0,00 \\
\hline $2,1(2)$ & $2,0(2)$ & $2,0(2)$ & 0,00 & $-0,5(2)$ & 0,00 \\
\hline $1,5(5)$ & $1,5(5)$ & $1,5(5)$ & 0,00 & $-0,1(5)$ & 0,00 \\
\hline $2,1(4)$ & $1,8(3)$ & $1,8(4)$ & $-0,1(3)$ & $-0,2(3)$ & $-0,2(3)$ \\
\hline
\end{tabular}

* Paramètres d'agitation thermique anisotrope / General displacement anisotropic parameters 


\section{TABLEAU III}

Distances et angles dans les polyèdres de cadmium.

Les atomes notés (') correspondent à des positions équivalentes.

Bond lengths and angles in cadmium polyhedra;

atoms noted (') correspond to equivalent positions.

\begin{tabular}{|c|c|c|c|c|c|}
\hline \multicolumn{2}{|c|}{ Polyèdre : $\mathrm{Cdl}(\mathrm{O} 1)_{2}(\mathrm{O} 2)_{2}(\mathrm{Cl})_{2}$} & \multicolumn{2}{|c|}{ Polyèdre : $\mathrm{Cd} 2(\mathrm{O} 1)(\mathrm{O} 2)_{2}\left(\mathrm{O}^{\prime}\right)_{2}(\mathrm{Cl})$} & \multicolumn{2}{|c|}{ Environnement autour de l'hydrogène } \\
\hline Distances $(\AA)$ & Angles $\left({ }^{\circ}\right)$ & Distances $(\AA)$ & Angles $\left({ }^{\circ}\right)$ & Distances $(\AA)$ & Distances $(\AA)$ \\
\hline$(2 X) \mathrm{Cd} 1-\mathrm{O} 1: 2,245(7)$ & $(1 X)$ O1-Cd1-O1': 180 & $(1 X) \mathrm{Cd} 2-\mathrm{O} 1: 2,328(11)$ & $(2 X)$ O1-Cd2-O2: $96,2(3)$ & $\mathrm{H} 1-\mathrm{O} 1: 0,90$ & $\mathrm{H} 2-\mathrm{O} 2: 0,92$ \\
\hline$(2 X) \mathrm{Cd} 1-\mathrm{O} 2: 2,241(8)$ & $(2 X)$ O1-Cd1-O2 : $80,8(3)$ & $(2 X) \mathrm{Cd} 2-\mathrm{O} 2: 2,247(8)$ & $(2 X)$ O1-Cd2-O2' : $78,5(3)$ & $\mathrm{H} 1-\mathrm{Cl}: 2,63$ & $\mathrm{H} 2-\mathrm{Cl}: 2,52$ \\
\hline$(2 X) \mathrm{Cd} 1-\mathrm{Cl}: 2,829(4)$ & $(2 X)$ O1-Cd1-O2': $99,2(3)$ & $(2 X) \mathrm{Cd} 2-\mathrm{O} 2 ': 2,268(8)$ & (1X) O1-Cd2-Cl : 178,2(3) & $\mathrm{O} 1-\mathrm{Cl}: 3,495(14)$ & $\mathrm{O} 2-\mathrm{Cl}: 3,421(9)$ \\
\hline & $(2 X)$ O1-Cd1-Cl : 96,5(3) & $(1 X) \mathrm{Cd} 2-\mathrm{Cl}: 2,735(4)$ & $(1 X)$ O2-Cd2-O2： 80,6(3) & & \\
\hline & $(2 X) \mathrm{O} 1-\mathrm{Cd} 1-\mathrm{Cl}^{\prime}: \quad 83,5(3)$ & & (2X) $\mathrm{O} 2-\mathrm{Cd} 2-\mathrm{O} 22^{\prime}: 174,6(3)$ & & \\
\hline & $(1 X) \mathrm{O} 2-\mathrm{Cd} \mathrm{l}-\mathrm{O} 2 ': 180$ & & $(2 X) \mathrm{O} 2-\mathrm{Cd} 2-\mathrm{O}^{\prime}:$ : 99,6(3) & & \\
\hline & $(2 X)$ O2-Cd1-Cl : $96,9(2)$ & & $(1 X) \mathrm{O}^{\prime}-\mathrm{Cd} 2-\mathrm{O} 2 ': 79,7(3)$ & & \\
\hline & $(2 X) \mathrm{O} 2-\mathrm{Cd} 1-\mathrm{Cl}^{\prime}: \quad 83,1(2)$ & & (2X) O2-Cd2-Cl : $85,2(2)$ & & \\
\hline & $(1 X) \mathrm{Cl}-\mathrm{Cd} 1-\mathrm{Cl}^{\prime}: 180$ & & $(2 X) \mathrm{O}^{\prime}-\mathrm{Cd} 2-\mathrm{Cl}: 100,2(2)$ & & \\
\hline
\end{tabular}





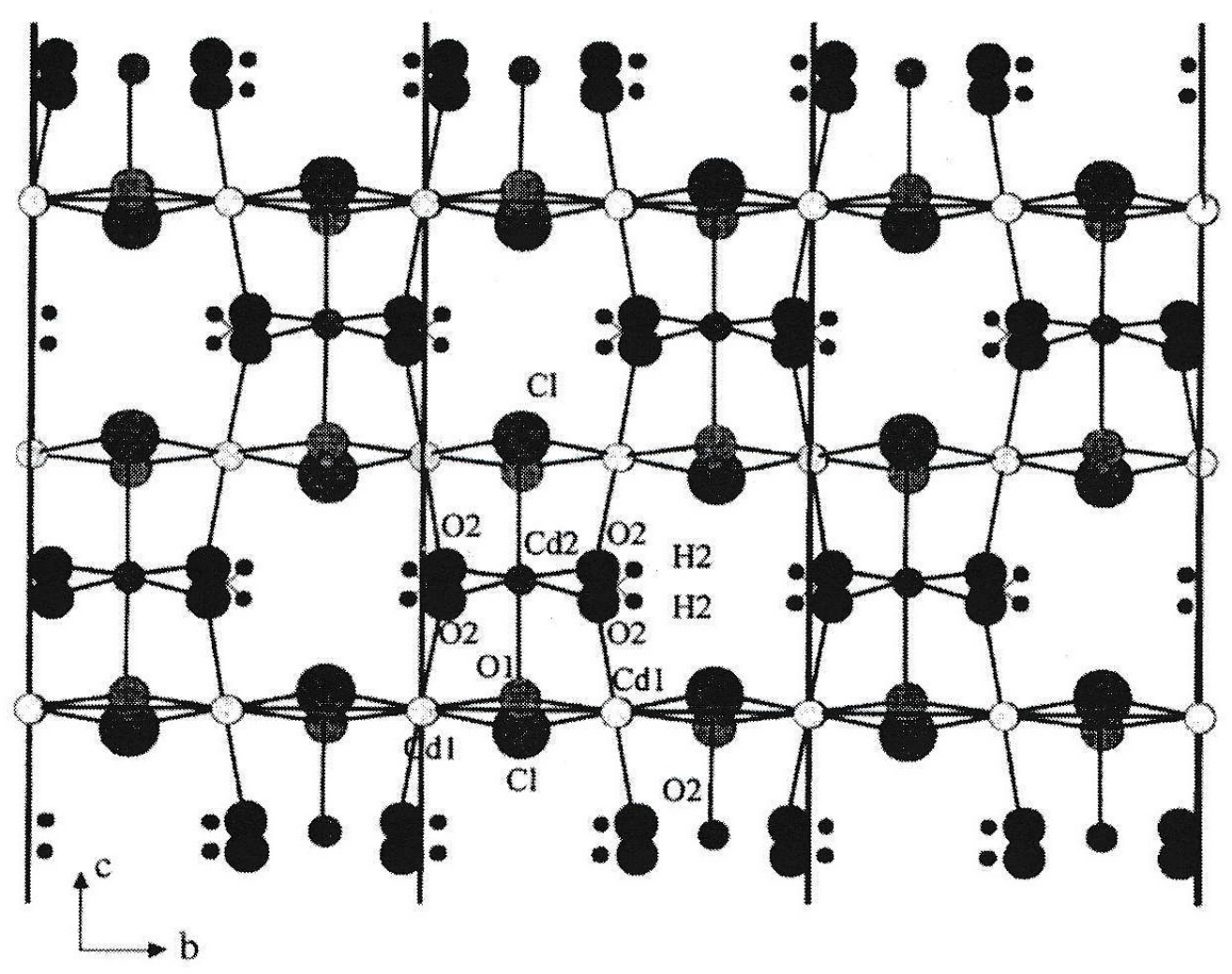

Fig. 1. - Projection de la structure suivant $\boldsymbol{a}$.

Fig. 1. - Structure projection along a.

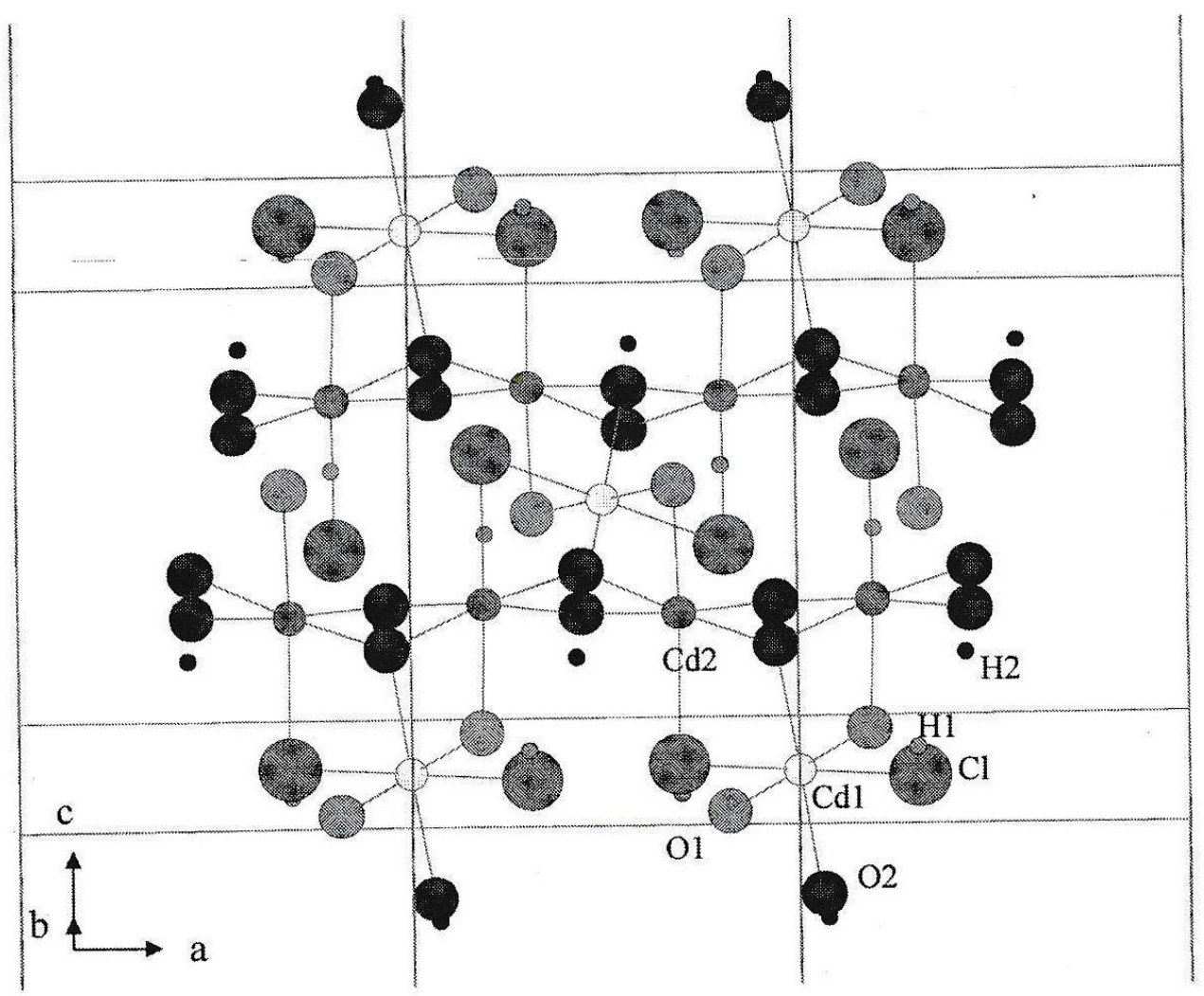

Fig. 2. - Vue en perspective.

Fig. 2. - Structure drawing in perspective. 

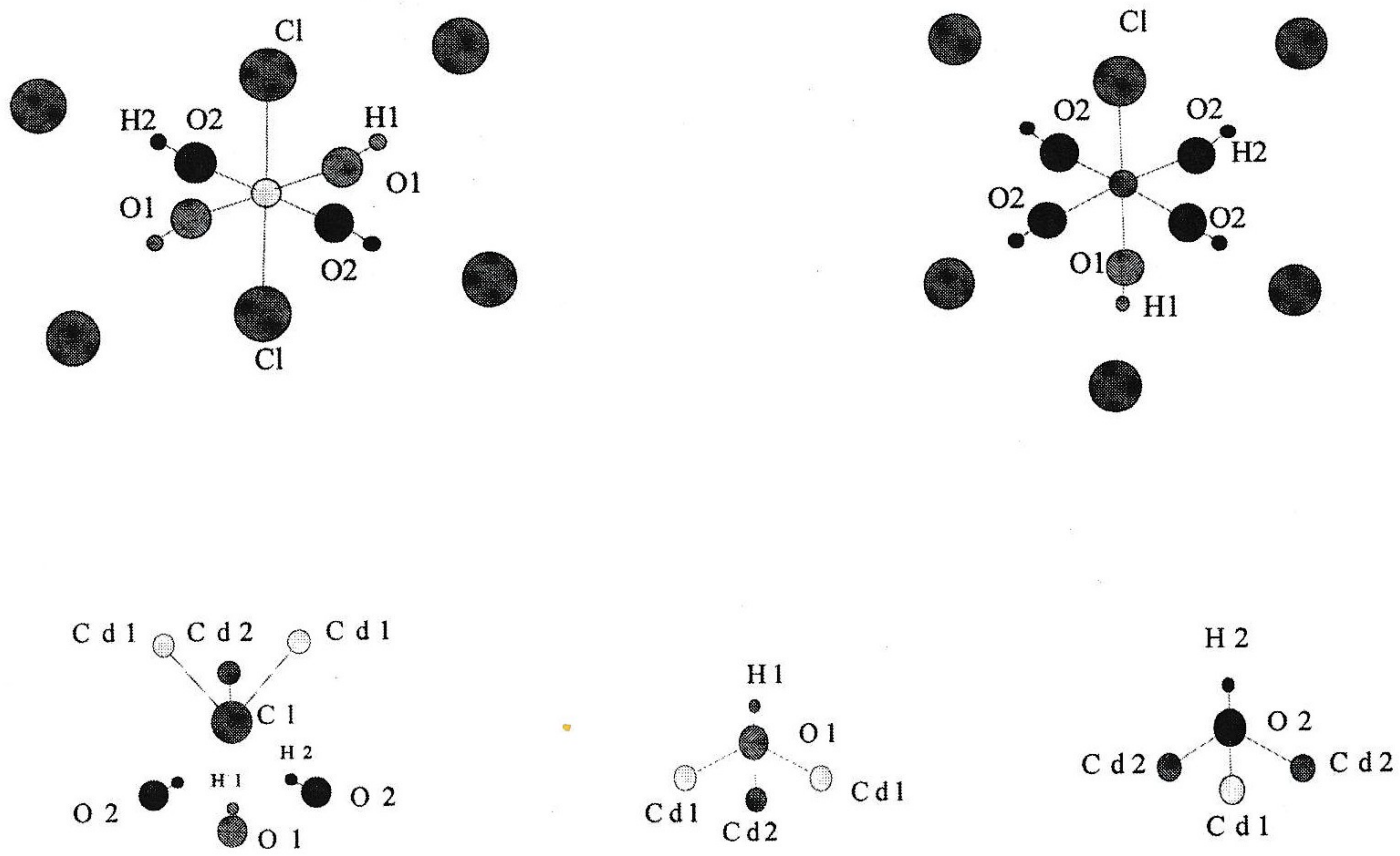

Fig. 3. - Environnement des atomes indépendants.

Fig. 3. - Surroundings of independent atoms. 